An International Journal of Optimization and Control: Theories \& Applications ISSN:2146-0957 eISSN:2146-5703

\title{
Generalized synchronization of identical and nonidentical chaotic dynamical systems via master approaches
}

\author{
Shko Ali-Tahir ${ }^{\text {a }}$, Murat Sarı ${ }^{* a}$, Abderrahman Bouhamidi ${ }^{b}$ \\ a Department of Mathematics, Faculty of Art and Sciences, Yildiz Technical University, Turkey \\ b L.M.P.A, Université du Littoral, 50 rue F. Buisson BP699, F-62228 Calais-Cedex, France \\ tahirshko@gmail.com,sarim@yildiz.edu.tr, bouhamidi@lmpa.univ-littoral.fr
}

\section{ARTICLE INFO}

\section{Article History:}

Received 03 July 2017

Accepted 13 October 2017

Available 25 October 2017

Keywords:

Dynamical system

Synchronization

Chaotic system

Lyapunov theory

Numerical analysis

\section{ABSTRACT}

The main objective of this work is to discuss a generalized synchronization of a coupled chaotic identical and nonidentical dynamical systems. We propose a method to study generalized synchronization in master-slave systems. This method, is based on the classical Lyapunov stability theory, utilizes the master continuous time chaotic system to monitor the synchronized motions. Various numerical simulations are performed to verify the effectiveness of the proposed approach.

AMS Classification 2010:

$34 D 06,37 D 45,34 D 45$

\section{Introduction}

For the last few decades, chaotic phenomena have been seen to be new kinds of oscillating system for the successful applications in different scientific fields including physics, chemistry, ecology, biology, etc. The chaotic oscillators are found in many dynamical systems of various origins. Their behavior is characterized by instability and limited predictability in time. The original work on synchronization was introduced in coupled pendulum by Huygens [1]. Since this discovery has been carried out, it has attracted very considerable attention over the past three decades in different scientific fields including physical and biological processes. The surprising synchronization phenomena generated between coupled chaotic systems has been discovered by Pecora and Carroll [2]. They proposed that "synchronization can be observed even in chaotic systems". Then, the synchronization of coupled chaotic systems has been extensively and intensively studied.
Thus, behavior of a chaotic phenomenon can be classified into several types of synchronization such as: identical or complete synchronization appears as the coincidence of states of interacting systems, phase synchronization which means entrainment of phases of chaotic oscillators in a closely controlled phase relationship, lag synchronization appears as having a parameter mismatch in mutually coupled chaotic oscillator. This type of lag synchronizations has important technological implications in engineering systems. In the literature, a large number of researchers have extensively concentrated on identical synchronization [2], generalized synchronization [3, 4], active control methods such as adaptive control, feedback control, sliding mode control, impulsive control and fuzzy control and so on [5-10].

In the case of synchronization of master-slave systems, the designed controller makes the trajectories of the state variables of the master system to track the trajectories of the slave system. This

*Corresponding Author 
fact may pose a trouble in using the results of theoretical analyses in practical applications of synchronized chaos.

In this paper, we further study a generalized synchronization of chaotic systems and propose a new and more general method for constructing a master system to implement the generalized synchronization with slave chaotic system by using the Lyapunov stability theory. This technique, known as master system method, is practically established in a simple form since it is effectively guaranteed for the stability of generalized chaotic synchronization. Besides, we present the generalized synchronization for two basic categories, namely; synchronization between two identical chaotic systems and synchronization between two nonidentical chaotic systems. Synchronization of nonidentical chaotic systems can be found in many natural systems, such as Lorenz system, Chua's system, Rossler system, asthalamic neurons in the human brain etc.

The rest of the paper is organized as follows: Section 2 gives a general description of a generalized synchronization by constructing master systems for detecting generalized synchronization of chaotic systems based on the classical Lyapunov stability theory. Section 3 studies the synchronization behaviour of two identical chaotic systems. Section 4 presents the results of an experiment with synchronization of nonidentical chaotic systems. Section 5 concludes main results of the present work and recommendations for further studies.

\section{Generalized synchronization systems}

The basic master and slave chaotic system is given as,

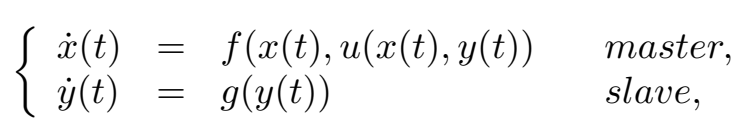

where the vector $x(t) \in \mathbb{R}^{n}$ and $y(t) \in \mathbb{R}^{n}$ represent the master signal and the slave signal, respectively. The functions $f \in R^{n}$ and $g \in R^{n}$ are

and

$$
f(x(t))=A x(t)+s(x(t))
$$

$$
g(y(t))=B y(t)+h(y(t))
$$

where $A$ and $B$ of size $n \times n$ are assumed to be constant matrices and the functions $s \in R^{n}$ and $h \in R^{n}$ represent the nonlinear parts of $f$ and $g$, respectively. The master system synchronizes with the system slave in a state of generalized synchronization, if there exists a transformation $u: \mathbb{R}^{2 n} \longrightarrow \mathbb{R}^{n}$, a manifold $T=\{(x, y) \in$ $\left.R^{2 n}, y(t)=\phi(x(t))\right\}$, where $\phi: \mathbb{R}^{n} \longrightarrow \mathbb{R}^{n}$ being a smooth function. The function transforms the trajectories on the attractor of the first system into those on the attractor of the second system. The problem consisting of the controller function $u$ exists and satisfies the following property:

$$
\lim _{t \longrightarrow \infty}\|e(t)\|=\lim _{t \longrightarrow \infty}\|y(t)-\phi(x(t))\|=0
$$

for all initial conditions.

To explain the synchronization of the driver and the response systems, the error equation is given by

$$
e(t)=y(t)-\phi(x(t))
$$

By the Lyapunov direct method we consider a Lyapunov function

$$
V(t)=\frac{1}{2} e(t)^{T} P e(t)
$$

Note that $P$ is a symmetric positive definite matrix and is independent of time. Here, $P$ is given as the identity matrix. We assume that the error system $e(t)$ is small enough and satisfies a differential equation of the form

$$
\dot{e}(t)=-M(t) e(t)
$$

where $M$ is an appropriate matrix.

In this paper, we construct a master system driven by the synchronization signal (4) to implement linear generalized synchronization with the slave system.

Now then

$$
\begin{aligned}
\dot{e}(t)= & \dot{x}(t)-\mathcal{J}_{\Phi}(y(t)) \dot{y}(t) \\
= & A x(t)+s(x(t))+u(x(t), y(t)) \\
& -\mathcal{J}_{\phi}(y(t)) f(x(t))
\end{aligned}
$$

where $\mathcal{J}_{\phi}$ is the Jacobian matrix of the function $\phi$. According to condition (4), the corresponding controller function $u$ exists and is given by

$$
\begin{aligned}
u(x(t), y(t))= & -M(t) e(t)+\mathcal{J}_{\phi}(y(t)) f(x(t)) \\
& -A x(t)-h(y(t)) .
\end{aligned}
$$

Then, system (1) becomes 


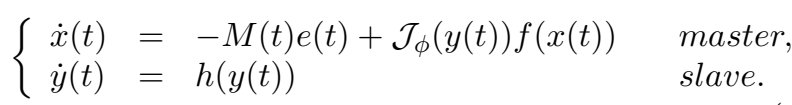

In order to find a generalized synchronization, the following assumptions on the master system are needed: $\Phi$ must be a continuously differentiable function and the matrix $M^{T}(t) P+P M(t)$ is a positive definite matrix. In the next section, we present two simulation results: first between two nonidentical systems and other between two identical systems. For the time integration the fourth order Runge-Kutta (RK4) method has been used under the initial conditions $x(0)$ and $y(0)$. The interval time $\left[t_{0}, T\right]$ is partitioned into $N$ subintervals $\left[t_{n}, t_{n+1}\right]$ with $t_{n}=t_{0}+n \Delta t$ for $n=0, \cdots, N$, $\Delta t=\frac{T-t_{0}}{N}$.

\section{Synchronization of two nonidentical systems}

The Rössler system described by the nonlinear autonomous differential equations is [11]:

$$
\left\{\begin{aligned}
\frac{d x_{1}}{d t} & =-y_{1}-z_{1}+u_{1}(x(t), y(t)) \\
\frac{d y_{1}}{d t} & =x_{1}+a y_{1}+u_{2}(x(t), y(t)) \\
\frac{d z_{1}}{d t} & =z_{1}\left(x_{1}-c\right)+b+u_{3}(x(t), y(t))
\end{aligned}\right.
$$

where, $u(t)=\left[u_{1}(1), u_{2}(t), u_{3}(t)\right]^{T}$ for $x(t)=$ $\left[x_{1}(t), y_{1}(t), z_{1}(t)\right], y(t)=\left[x_{2}(t), y_{2}(t), z_{2}(t)\right]^{T}$. The set of parameters representing chaotic attractors for the famous Rössler systems are taken to be $a=0.25, b=3, c=0.5$ and $d=0.05$ and chosen as master system.

The unified chaotic system is described as follows [12]:

$$
\left\{\begin{array}{rl}
\frac{d x_{2}}{d t} & =(25 \alpha+10) x_{2}-(25 \alpha+10) y_{2} \\
\frac{d y_{2}}{d t} & =(28-35 \alpha) x_{2}+(29 \alpha-1) y_{2}-x_{2} z_{2} \\
\frac{d z_{2}}{d t} & =-\frac{(\alpha+8}{3} z_{2}+x_{2} y_{2}
\end{array} .\right.
$$

Thus system (9) reduces to the Lorenz system if $\alpha=0$ and chosen as the slave system. The
Rössler and Lorenz systems exhibit the chaotic attractor with the initial conditions are chosen to be $x(0)=(1,1,1)^{T}$ and $y(0)=(1,1,0)^{T}$. The time interval is taken to be $\left[t_{0}, T\right]=[0,2000]$ as shown in Figure 1.

To achieve the reduced order synchronization behavior between the Rössler and Lorenz systems, we consider that the Rössler as the master system and Lorenz as the slave system. Then, we rewrite the master system in the form

$$
\dot{y}=-M(t) e(t)+\mathcal{J}_{\Phi}(y(t)) f(x(t)),
$$

where

$$
B=\left[\begin{array}{ccc}
0 & -1 & -1 \\
1 & a & 0 \\
0 & 0 & -c
\end{array}\right] \text { and } g(y)=\left[\begin{array}{c}
0 \\
0 \\
x_{1} z_{1}+b
\end{array}\right]
$$

To solve the synchronization problems of (8) and (9) with the control functions $u(x(t), y(t))$ as calculated by (6), one can define the nonidentity vector function as $\Phi\left(x_{1}, y_{1}, z_{1}\right)=\left(x_{1}+z_{1}, y_{1}+\right.$ $\left.z_{1}, x_{1}\right)^{T}$. For the sake of the simplicity, we consider $M=k I_{3}$. We see that the master-slave systems are synchronized by the designed controller function between two nonidentical chaotic systems, namely, that $\lim _{t \longrightarrow \infty}\left|x_{2}-x_{1}\right|=0, \lim _{t \longrightarrow \infty} \mid y_{2}-$ $y_{1} \mid=0$ and $\lim _{t \rightarrow \infty}\left|z_{2}-z_{1}\right|=0$. Thus, we confirm that the convergence of the synchronization is obtained at the optimal value $k \bumpeq 0.7$ as shown in Figure 2,

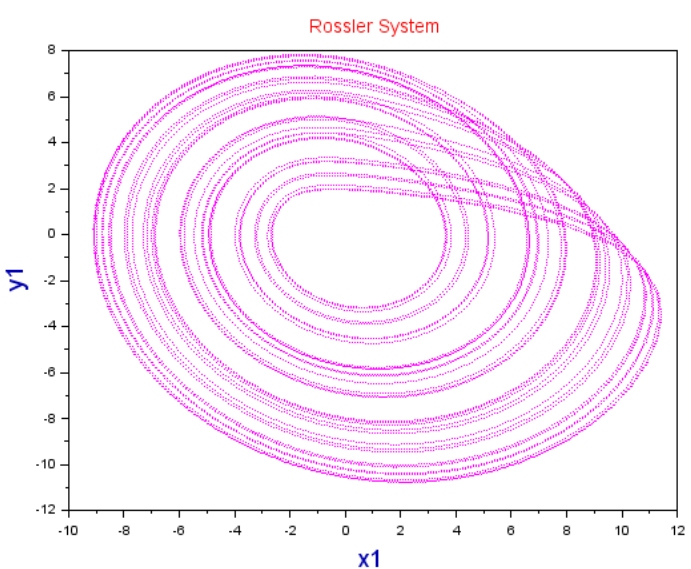




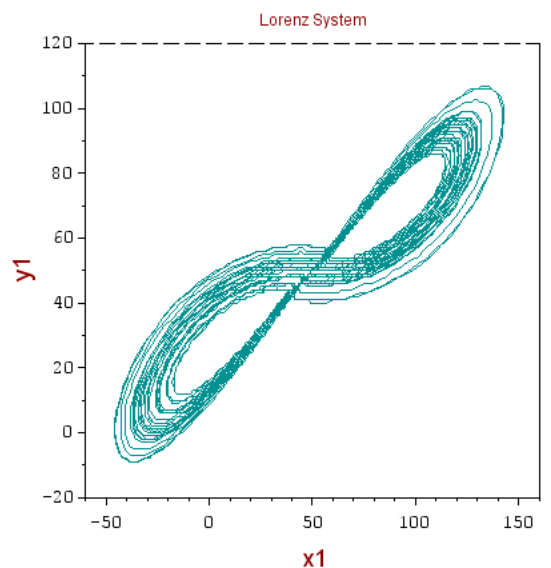

Figure 1. The chaotic attractor of the Rössler and Lorenz systems: $x_{1} x_{2^{-}}$plane projections.

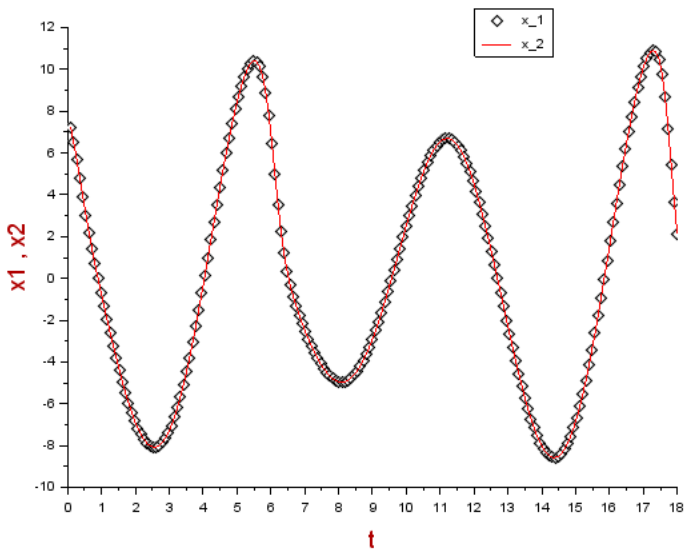

Figure 2. Time series for $x_{2}$ and $x_{1}$ for the coupling strengths $k=$ $0.1,0.4,0.7$, respectively.

\section{Synchronization of two identical systems}

In this section, the proposed method is applied to synchronization behaviour of two identical Hindmarsh-Rose (HR) neuron systems. We fo-

$$
\left\{\begin{aligned}
\frac{d x_{1}}{d t}= & -a z_{1}+b x_{1}^{2}+c x_{1}+d+e y_{1} \\
& -f x_{1}^{3}+g I(t)+u_{1}(x(t), y(t)) \\
\frac{d y_{1}}{d t}= & -\alpha-\beta x_{1}^{2}-\mu y_{1}-\gamma x_{1} \\
& +u_{2}(x(t), y(t)) \\
\frac{d z_{1}}{d t}= & \eta\left(\rho\left(x_{1}-C\right)-z_{1}\right) \\
& +u_{3}(x(t), y(t))
\end{aligned}\right.
$$
cus on the synchronisation of two well defined chaotic systems in which the control method can be applied to the master-slave synchronization of the HR neurons. The HR model is then given by 13,14 .
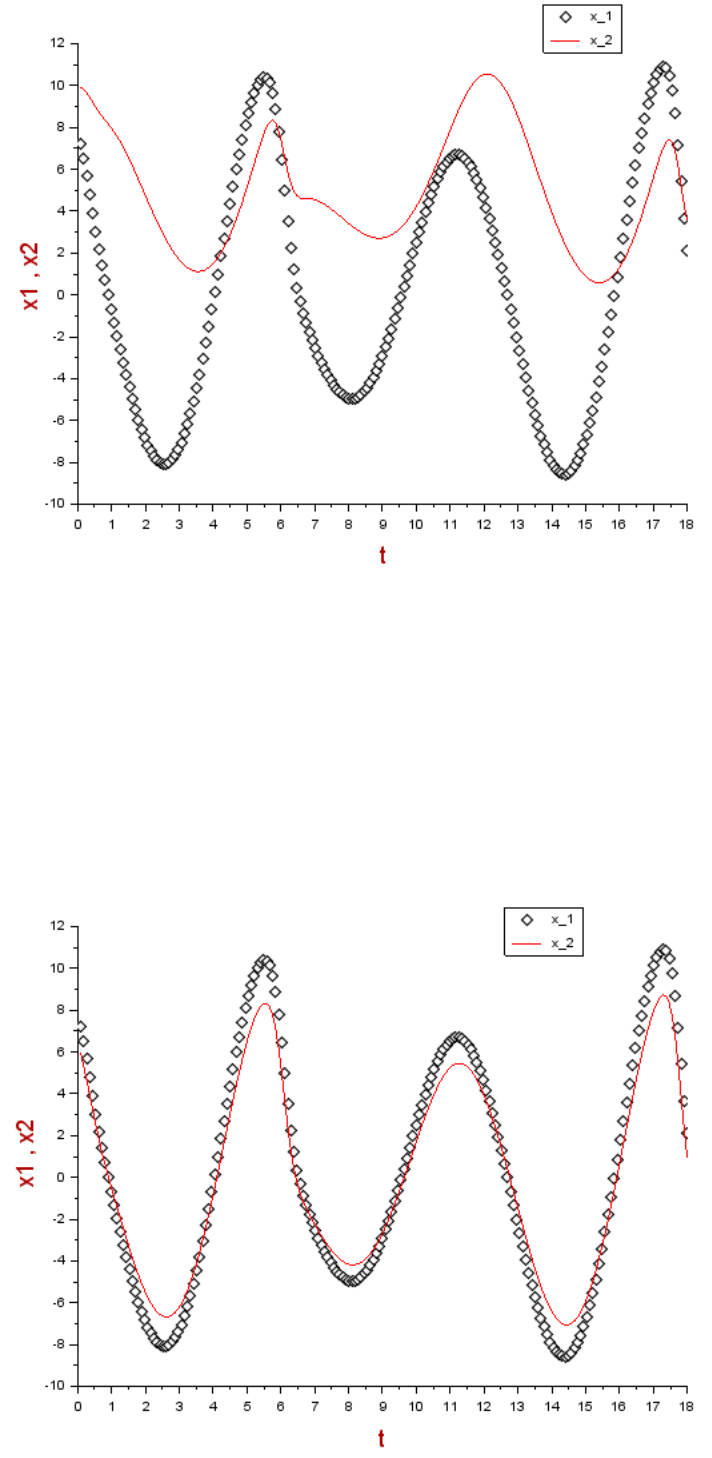

where, $u(t)=\left[u_{1}(1), u_{2}(t), u_{3}(t)\right]^{T}$ for $x(t)=$ $\left[x_{1}(t), y_{1}(t), z_{1}(t)\right], y(t)=\left[x_{2}(t), y_{2}(t), z_{2}(t)\right]^{T}$. The set of parameters are given as $a=1, b=0$, $c=3, d=2, e=5, f=1, g=1, \alpha=1, \beta=1$, $\mu=2, \gamma=1, \eta=0.005, \rho=4$ and $C=-2.6$. Here, $I(t)$ is the externally applied current at time $t$ and the parameter $C$ is the $x$-coordinate of the leftmost equilibrium point of the model without adaptation. To study synchronization motions of the two identically coupled HR neuronal systems, 
it is assumed that system (10) is considered to be the master system. The slave system is given by

$$
\left\{\begin{array}{rl}
\frac{d x_{2}}{d t}= & -a z_{2}+b x_{2}^{2}+c x_{2}+d+e y_{2} \\
& -f x_{2}^{3}+g I(t) \\
\frac{d y_{2}}{d t}= & -\alpha-\beta x_{2}^{2}-\mu y_{2}-\gamma x_{2} \\
\frac{d z_{2}}{d t}= & \eta\left(\rho\left(x_{2}-C\right)-z_{2}\right)
\end{array} .\right.
$$

We can rewrite system (10) as

$$
\dot{y}(t)=-M(t) e(t)+\mathcal{J}_{\Phi}\left(X_{2}(t)\right) f\left(X_{2}(t)\right),
$$

where

$B=\left[\begin{array}{ccc}c & e & -a \\ -\gamma & -\mu & 0 \\ \eta \rho & 0 & -\eta\end{array}\right]$ and $g(y)=\left[\begin{array}{c}b x_{2}^{2}-f x_{2}^{3}+g I \\ -\alpha-\beta x_{2}^{2} \\ -C(\eta \rho)\end{array}\right]$

Again, as in the above example, the control functions $u(x(t), y(t))$ are given by (6), where $\Phi\left(x_{1}, y_{1}, z_{1}\right)=\left(x_{2}, y_{2}, z_{2}\right)$ is the identity function. We produce numerical results using the RK4 method for the master-slave systems, by using $M=k I_{3}$. In our simulation, the initial conditions are chosen to be $x(0)=(-0.54,-1,3)^{T}$ and $y(0)=(0.54,1,-3)^{T}$. The time interval is taken to be $\left[t_{0}, T\right]=[0,5000]$. The chaotic behavior of the HR system is shown in Figure 3. for $\mathrm{I}=3.25$. In Figure 4, we consider coupling for $k \longrightarrow \infty$. It can be seen that synchronization occurs for $k \bumpeq 0.2$. Thus, all the variables of the coupled chaotic systems converge, namely, $x_{2}$ converges to $x_{1}, y_{2}$ to $y_{1}$, and $z_{2}$ to $z_{1}$. Thus, we can see the generalized synchronization between the master and slave chaotic systems with the designed controller functions.

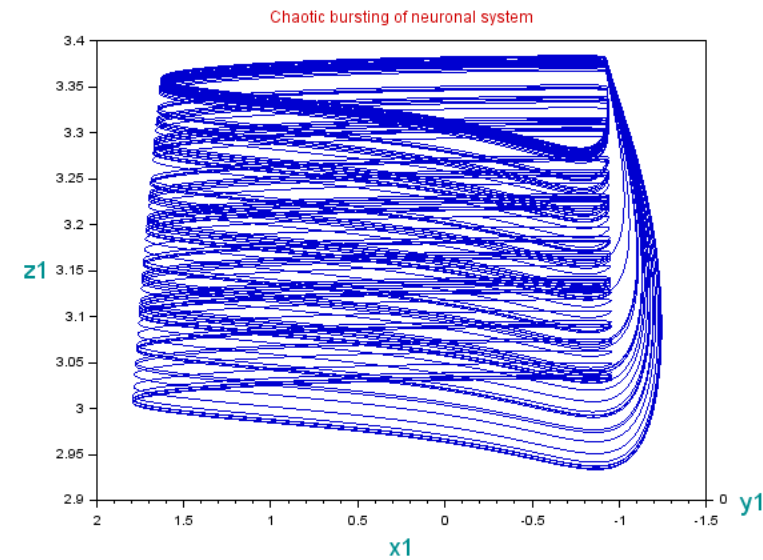

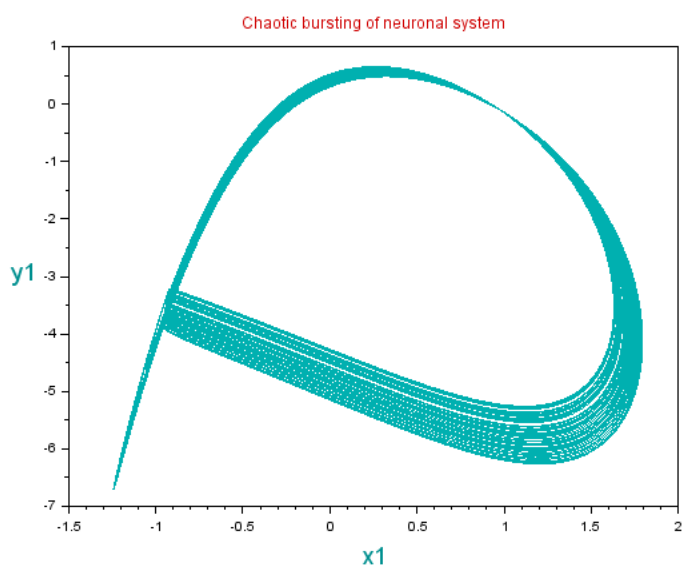

Figure 3. Chaotic attractors of the HR system: $x_{1} x_{2}-$ plane projections.
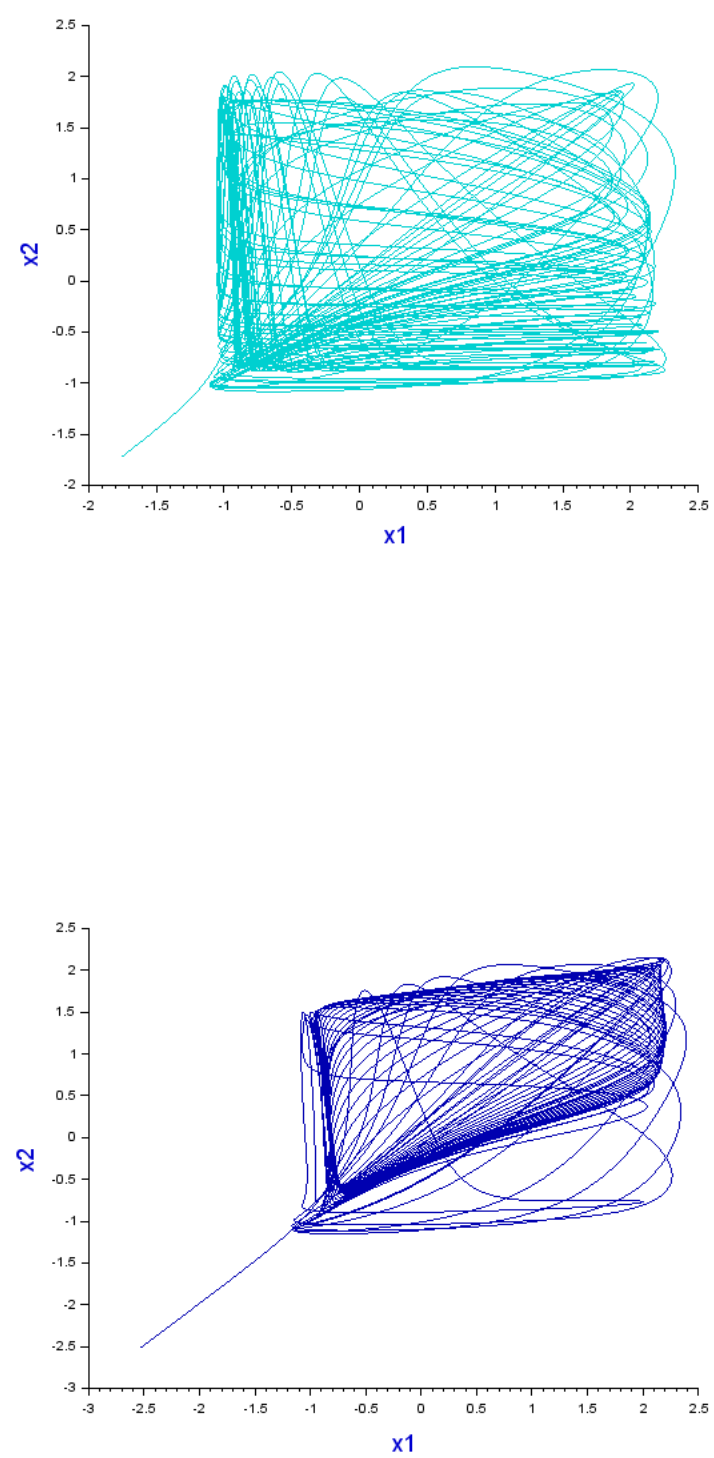

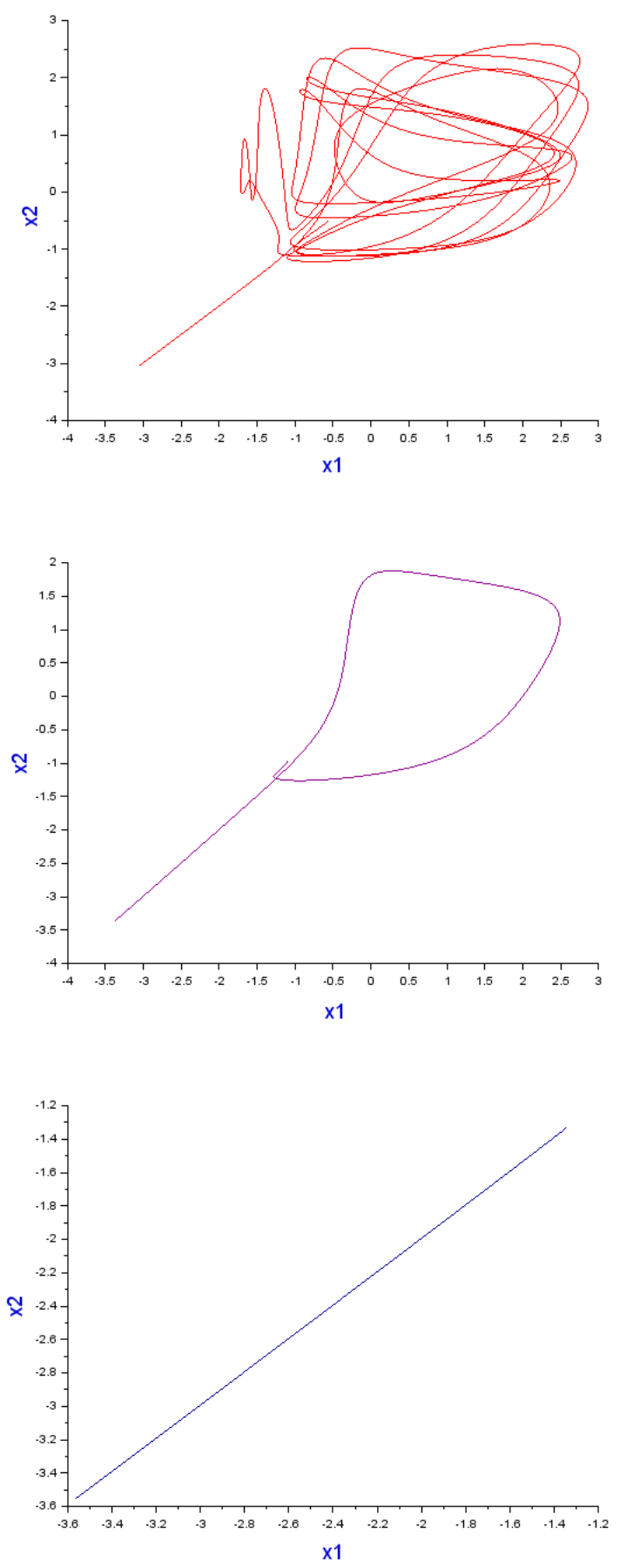

Figure 4. Synchronization between the master-slave HR neurons systems: plots of $x_{2}$ according to $x_{1}$ at various coupling strengths $k=$ $0.05,0.08,0.17,0.19$ and 0.2 .

\section{Conclusion}

In this paper, the generalized synchronization of spatial chaotic identical and nonidentical dynamical systems have been analysed. We have reconstructed the master system based on the classical Lyapunov stability theory. The designed controllers enable the state variables of the slave system to globally synchronize the state variables of the master system in both physical and biological systems. It should be pointed out that the present method has effectively been extended to generalized synchronization. The present method has also provided the guaranteed stability of the error dynamics at the origin . All computations have been carried out in terms of the codes produced here in SCILAB-6.0. For further the theory of synchronization stays a challenging problem of nonlinear behavior.

\section{Acknowledgments}

The first author would like to express his gratitude to the University of Sulaimani. His thanks also goes to the Turkish government scholarships for the International students (YTB) that supported him financially throughout his $\mathrm{PhD}$ studies.

\section{References}

[1] Huygens, (1973). Horoloquim oscilatoruim apud f.muquet,parisiis, The pendulum clock, Ames:Iowa state university press, (English translation).

[2] Pecora, L.M., Carroll, T.L. (1990). Synchronization in chaotic systems. Phys Rev Lett., 64, 821-824.

[3] Gonzalez-Miranda, J.M. (2002). Generalized synchronization in directionaly coupled systems with identical individual dynamics. Phys Rev E, 65, 047202-4.

[4] Kocarev, L., Partlitz, U., (1996). Generalized synchronization predictability and equivalence of undirectionally coupled dynamical systems. Phys Rev Lett., 76, 1816-1819.

[5] Du, H.Y., Shi, P., Lu, N. (2013). Function projective synchronization in complex dynamical networks with time delay via hybrid feedback control. Nonlinear Anal. Real., 14, 1182-90.

[6] Jin, X.Z., Yang, G.H. (2013). Adaptive sliding mode fault-tolerant control for nonlinearly chaotic systems against network faults and time-delays. J. Franklin Inst., 350, 1206-1220.

[7] Mei, J., Jiang, M.H., Xu, W.M., Wang, B. (2013). Finite-time synchronization control of complex dynamical networks with time delay. Commun Nonlinear Sci Numer Simulat., 18, 2262-478.

[8] Quiroga, R.Q., Arnhold, J., Grassberger, P. (2000). Learning driver-response relationships from synchronization patterns. Physical Review E, 61, 5142-5148.

[9] Tahir, S.A., (2013). The synchronization of identical Memristors systems via Lyapunov direct method. Applied and Computational Mathematics, 6, 130-136.

[10] Zhou, J.P., Wang, Z., Wang, Y., Kong, Q.K. (2013). Synchronization in complex dynamical networks with interval time-varying coupling delays. Nonlinear Dyn., 72, 2487-2498.

[11] Peitgen, H-O., Jürgens, H., Saupe, D. (1992). Chaos and fractals, Springer-Verlag.

[12] Ahmada, I., Bin Saabana, A., Binti Ibrahima, A., Shahzadb, M., Naveedc, N. (2016). The synchronization of chaotic systems with different dimensions bya robust generalized active control. Optik, 127, 8594871. 
[13] Hindmarsh, J.L., Rose, R.M. (1984). A model for neuronal bursting using three coupled differential equations. Proc. R. Soc. Lond., , 221, 87-102.

[14] Hrg, D. (2013). Synchronization of two HindmarshRose neurons with unidirectional coupling. Neural Netw., 40, 73-79.

Shko Ali-Tahir is currently working on a doctorate in applied mathematics at Yildiz Technical University in Turkey. He completed his master in mathematics and computer science of complex and distributed systems at Le Havre university in France. Since 2011, he has been the assistance lecture at Sulemani University in Iraq. His research interests include Dynamical systems, Partial differential equations, Spline methods and meshfree methods.
Murat Sar is an Associate Professor in the Department of Mathematics at Yildiz Technical University, Istanbul, Turkey. He received his BSc degree from Ondokuzmayis University, Turkey in 1991. He completed his graduate studies (MSc/PhD degrees) in Mathematics from University of South Wales, UK in 2000. His research interests are numerical methods, wave propagation, computational methods, mathematical modelling, biophysical modelling, medical modelling and economical modelling.

Abderrahman Bouhamidi is a Professor in the Department of Mathematics at the Littoral University, Calais France. He received his BSc/MSc degree from Lille University, France in 1990 and his Phd in Mathematics from University of Nantes, France in 1992. His research interests are numerical analysis, approximation theory, optimization and linear algebra.

An International Journal of Optimization and Control: Theories \& Applications (http://ijocta.balikesir.edu.tr)

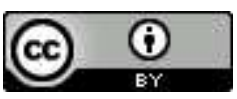

This work is licensed under a Creative Commons Attribution 4.0 International License. The authors retain ownership of the copyright for their article, but they allow anyone to download, reuse, reprint, modify, distribute, and/or copy articles in IJOCTA, so long as the original authors and source are credited. To see the complete license contents, please visit http://creativecommons.org/licenses/by/4.0/. 